\title{
Compensatory growth in novel Drosophila Akt1 mutants
}

\author{
Jennifer D Slade and Brian E Staveley
}

\begin{abstract}
Background: Organisms, tissues and cells are genetically programmed to grow to a specific largely pre-set size and shape within the appropriate developmental timing. In the event of mutation, cell death, or tissue damage, the remaining cells may increase their rate of growth to compensate and generate an intact, potentially smaller, tissue or organism in order to achieve the desired size. A delay in the developmental timing could aid in this process. The insulin receptor signalling pathway with its central component, the Akt1 kinase, and endpoint regulator, the transcription factor foxo, plays a significant role in the control of growth. Drosophila melanogaster is an excellent model organism with a well-studied life cycle and a consistently developing compound eye that can undergo analysis to compare changes in the properties of adult ommatidia as an indicator of growth.

Findings: Imprecise excision of a PZ P-element inserted in the upstream region of Akt1 generated several novel hypomorphic alleles with internally deleted regions of the Pelement. These mutations lead to small, viable Drosophila that present with delays in development. Suppression of this phenotype by the directed expression of $A k t 1^{+}$indicates that the phenotypes observed are Akt1 dependent. Somatic clones of the eyes, consisting of homozygous tissue in otherwise heterozygous organisms that develop within a standard timeframe, signify that more severe phenotypes are masked by an extension in the time of development of homozygous mutants. Generation of Drosophila having the hypomorphic Akt1 alleles and a null allele of the downstream target foxo result in a phenotype very similar to that of the foxo mutant and do not resemble the Akt1 mutants.

Conclusion: The developmental delay of these novel Akt1 hypomorphs results in a latent phenotype uncovered by generation of somatic clones. The compensatory growth occurring during the extended time of development appears to be implemented through alteration of foxo activity. Production of clones is an effective and informative way to observe the effects of mutations that result in small, viable, developmentally delayed flies.
\end{abstract}

\section{Background}

The cell is the basic structural unit of all living organisms. The overall size of a cell can either augment or limit its ability to perform essential functions. Consequently size homeostasis is pertinent for the fitness and function of cells. Even slight disruption of this homeostasis can lead to disease, thus it is critical to understand the complex mechanisms that control cell growth. Drosophila melanogaster develops quickly through a sequence of three feeding and growing larval stages followed by pupation and eclosion [1] and is an ideal model system in which to study cell growth.

\footnotetext{
* Correspondence: bestave@mun.ca

Department of Biology, Memorial University of Newfoundland, 232 Elizabeth Avenue, St. John's, Newfoundland and Labrador A1B 3X9, Canada
}

A crucial point in the control of growth in Drosophila is the achievement of the critical mass, the minimum weight required for transition from larvae to pupae, upon which any further feeding, or lack of feeding, will not prevent this change [2,3]. Drosophila larvae, when fed generously, can grow to, or past, the critical weight within four days. Restriction of dietary proteins slows this process, while total absence can halt growth completely [4]. Once larvae have reached the critical weight required for pupation, they may continue to feed for a period of time before undergoing the transition [5]. Several factors can influence the rate of growth during the larval stages including nutrition, temperature, density of organisms present in the environment, and underlying genetic mechanisms [6-10]. Slowed growth, due to genetic mechanisms or nutrient conditions, characteristically 
results in larvae that develop into smaller adults. While many mutations can influence growth; some alter the growth of individual organs, some retard overall growth without changing the final adult size, the mutations which slow growth and lead to a reduction in the overall organ and body size may be the most intriguing.

The conserved insulin receptor (InR) signalling pathway is implicated in the management of final adult size. In Drosophila, this highly conserved pathway has been shown to control cell size and growth, and to regulate body size and nutrient usage $[11,12]$. When any of the seven Drosophila insulin-like peptide (Ilp) genes are overexpressed, growth rates in larvae and adults are greatly increased, and ablation of the medial neurosecretory cells in the brain (the main producer of Ilps) leads to a decrease in the growth rate and final size [13]. Overexpression of the upstream components of the pathway, including the ligand (Ilps), the insulin receptor (Inr) and the insulin receptor substrate (chico), in Drosophila results in larger than normal flies, while mutation or loss of function of these components results in size reduction and developmental delay [14]. This reinforces the pivotal role of insulin receptor signalling in the control of growth.

The Akt1 kinase is a central component of the insulin receptor signalling pathway. When Drosophila Akt1 is overexpressed, it is shown to increase cell size but not proliferation, or number of cells, by overriding the control mechanisms that are responsible in determining the final size of cells [15]. Loss of $A k t 1$ can result in lethality [16] while hypomorphic activity can result in the production of smaller adults [15]. A key downstream target of Akt1, the transcription factor foxo, mediates the transcriptional regulation of the insulin pathway and controls several important cellular functions including metabolism, cell cycle regulation, DNA repair, apoptosis and protection of the cell against oxidative stress [17-20]. Through these diverse functions, the transcription factor foxo can facilitate the end result of Akt11 activity upon the regulation of cell growth and survival.

In order to explore the influence of $A k t 1$ activity upon cell growth, a series of novel Akt1 hypomorphs were generated through imprecise excision of a P-element situated in the control region upstream of the gene's coding region. A subset of these hypomorphs were selected, based upon reduction in adult size, and were further characterized with replacement analysis to confirm the reduction in size was due to altered $A k t 1$ activity. Due to the extended time required to reach eclosion by the homozygotes, somatic clones of the eye were generated to produce a mutant phenotype less influenced by a developmental delay. Finally, to further investigate the dynamic interaction between $A k t 1$ and foxo, Drosophila lines with novel hypomorphic alleles and a null version of the downstream target foxo gene were created and the potential for epistasis was evaluated. Our intent through these experiments is to better understand the effect of extended development time upon the overall phenotype of the novel Akt1 hypomorphs.

\section{Findings \\ Methods \\ Drosophila stocks, media and culture}

The initial P-element insertion line $r y^{506} P\{P Z\} A k t 11^{04226}$, TM3, $r y^{R K}, \mathrm{Sb}^{1}, \mathrm{Ser}^{1}\left(A k t 1^{04226}\right)$ was obtained from the Bloomington Drosophila Stock Center. This line contains a P-element inserted within the $5^{\prime}$ untranslated region of the $A k t 1$ gene on the third chromosome. Initial reports of this allele state that it is semi-lethal [21], but we, in addition to other researchers $[22,23]$ have found the homozygotes to be viable. The control line $w^{1118}$; P[FRT; $\left.w^{+}\right]^{2 A} P\left[r y^{+} n e o^{R} F R T\right]^{82 B} A k t 1^{+}$was derived from lines obtained from Norbert Perrimon of Harvard University. The $P \Delta 2-3, r y^{+}$line was utilized to generate the novel $A k t 1$ mutants [24]. The foxo null mutant line $w ;$ foxo ${ }^{W 124 X}$ was obtained from Drs. E. Hafen and M. Junger [25] of the University of Zurich. Wild-type Oregon R (OrR) stock was obtained from the Bloomington Drosophila Stock Center and $w^{1118}$ was obtained from Dr. Howard Lipshitz from the University of Toronto. Stocks and crosses were maintained on a standard medium containing cornmeal, molasses, yeast, agar and water. Routinely, stocks were kept at room temperature $\left(22 \pm 2^{\circ} \mathrm{C}\right)$ while crosses and experiments were carried out at $25^{\circ} \mathrm{C}$.

\section{Generation of Drosophila lines}

Hypomorphic alleles of $A k t 1^{04226}$ were generated via P-element excision by crosses to a line containing a stable source of transposase, $P \Delta 2-3$. The critical class offspring of the dysgenic males and $L y / T M 3, S b r y$ females were selected based upon loss of the $P Z$ P-element by the presentation of the rosy eye colour phenotype. These novel alleles were expected to differ from the $A k t 1^{+}$line and $A k t 1^{04226}$ by the resultant alterations of the PZ P-element and/or the adjacent Akt1 sequences. To allow for clonal analysis, recombinants of $w ; P\left[F R T ; w^{+}\right]^{2 A} P\left[r y^{+} n e o^{R} F R T\right]^{82 B}$ and the novel derivatives of $A k t 1^{04226}$ were generated and balanced over $T M 6 B, H u T b e$. Of the derivatives generated, a subset of these recombinants were selected for analysis based on the appearance of non-Tubby homozygotes.

Replacement studies were carried out by generating independent lines of $w^{1118}$; UAS-Akt1 $1^{+} / C y O ; A k t 1^{m}$ / TM6B and $w^{1118}$; arm-GAL4/CyO; Akt $1^{m} / T M 6 B$ where $m$ represents each of the novel $A k t 1$ mutant alleles. Crosses between these lines generated the critical class of $w^{1118}$; UAS-Akt1 $1^{+} /$arm-GAL4; Akt $1^{m} / A k t 1^{m}$ to be analyzed. 
The presence of FRT sites near the centromere of the 3R chromosome arm in the $A k t 1^{04226}$ derivative stocks allowed for somatic clones to be generated. The Drosophila line $y w ; P\left\{w^{+m}=\text { GAL4-ey. } H\right\}^{3-8} P\left\{w^{+m C}=U A S\right.$ FLP1.D $\}^{J D 1} ; P\left\{r y^{+t 7.2}=n e o F R T\right\}^{82 B} P\left\{w^{+m C}=\text { GMR-hid }\right\}^{S S 4}$ $l(3) C L-R^{1} / T M 2$ possesses eyeless-driven FLP and a distal recessive lethal allele [26] which, when crossed to each of the $A k t 1^{04226}$ derivatives generated the critical class of $y$ w; $P\left\{w^{+m}=\text { GAL4-ey. } H\right\}^{3-8} \quad P\left\{w^{+m C}=U A S-F L P 1 . D\right\}^{J D 1} /+$; $P\left[F R T ; w^{+}\right]^{2 A} \quad P\left[r y^{+} \quad n e o^{R} \quad F R T\right\}^{82 B} \quad A k t 1^{m} / P\left\{r y^{+t 7.2}=\right.$ neoFRT $\}^{82 B} \quad P\left\{w^{+m C}=G M R \text {-hid }\right\}^{S S 4} l(3) C L-R^{1}$ where $m$ represents the allele of $A k t 1$ derived from $A k t 1^{04226}$. The distal lethal allele resulted in the death of any homozygous $A k t 1^{+}$cells thereby making the eye almost completely composed of homozygous $A k t 1^{m}$ cells.

Generation of flies bearing both the novel mutant Akt1 alleles and a null foxo allele was performed via standard recombinant methods. As $A k t 1^{52}$ and $A k t 1^{57}$ exhibited the greater developmental delay, these alleles were selected for recombination with the null allele of foxo. From these combinations, a series of lines were selected based upon adult phenotypes and confirmed through PCR and sequencing.

\section{Molecular characterization of the novel hypomorphs}

Homozygous wild type, novel hypomorphic and double mutant Drosophila samples were collected from crosses of adult heterozygous female virgins to heterozygous males of each genotype. Ten homozygous male flies were collected upon eclosion and aged three to five days before being flash-frozen at $-70^{\circ} \mathrm{C}$. DNA was extracted from each sample via a standard phenolchloroform protocol. The Flybase database (http://flybase.org) includes the complete sequence for the $\mathrm{PZ}$ P-element positioned within the $A k t 1^{04226}$ line, and the National Center for Biotechnology Information (NCBI) database (http://www.ncbi.nlm.nih.gov/) includes the complete gene sequence for $A k t 1^{+}$. To design oligonucleotides indicating the breakpoint region of each novel mutant, a series of oligonucleotides both flanking and spanning the P-element insertion site was carried out via Primer3. PCR analysis via primers spanning the PZ Pelement revealed forward primer sites present and positioned near the breakpoints. A reverse primer positioned near the end of the $\mathrm{PZ}$ P-element allowed for generation of breakpoint fragments using HotStart Taq Polymerase (Qiagen Inc.) in an Eppendorf Mastercycler gradient thermal cycler through standard methods. Gels were photographed with a ChemilmageTM Ready 4400 v5.5 photodocumentation system. Purification and sequencing of the PCR products was completed at the Genomics and Proteomics (GaP) facility, Memorial University of Newfoundland.

\section{Analysis of developmental timing}

Heterozygotes are identified based upon the presence of Humeral (Hu), an allele of Antennapedia carried by the $T M 6 B, H u T b$ e balancer chromosome that results in extra bristles on the outer edges of the prothorax, while homozygous mutant flies lack this marker. Heterozygous females and males of the novel Akt1 hypomorphs were transferred to fresh media, incubated at $25^{\circ} \mathrm{C}$ for six hours to allow for egg-laying then removed. Vials were returned to the incubator immediately after removal of adult flies and examined each morning for fifteen days. Observations included days until pupation and eclosion. Pupae and adult flies were scored as Tubby heterozygotes or non-Tubby mutants and used to generate developmental delay line graphs in GraphPad Prism Version 5.03.

\section{Biometric analysis of Drosophila eyes}

Critical class males of the homozygous mutant Akt1 alleles, the transgenic rescues, the somatic clones and the double mutant lines were collected and aged for three days. Flies were then flash-frozen at $-70^{\circ} \mathrm{C}$ before preparation for scanning electron microscopy. Preparation included mounting upon aluminum SEM studs, desiccated and sputter coating in gold. Images were taken with either a Hitachi S-170 or S-570 Scanning Electron Microscope as per standard methods and analyzed using NIH Image J software [27].

\section{Results}

\section{Three novel Akt 1 mutants retain portions of inserted PZ P-element}

Molecular characterization of the three small viable $A k t 1$ mutants revealed internally deleted versions of the $P Z$ P-element at the original point of insertion (Figure 1). Analysis indicates that the retained sections are from both ends of the PZ P-element. Akt1 $1^{87}$ possesses the largest deleted region (11029 base pairs) between nucleic acids 2184 (within lacZ) and 13213 (within $r y^{+}$) of the PZ P-element sequence. The next largest deletion (9532 base pairs) is in $A k t 1^{52}$ between nucleic acids 2754 (within lacZ) and 12307 (within $r y^{+}$). Lastly $A k t 1^{57}$ has the smallest deletion of the three mutants of 3259 base pairs between nucleic acid 4315 (within the HSP70 polyadenylation control region) and 7574 (within $r y^{+}$). Each deletion includes a part of the $r y^{+}$gene, responsible for the phenotype $\left(r y^{-}\right)$upon which these mutants were selected. No alteration to the $A k t 1$ coding region sequence was detected in the three mutants.

\section{Three novel Akt1 mutants are developmentally delayed}

In the development from embryo to adult, $A k t 1$ mutant heterozygotes are similar to controls (Figure 2). The formation of heterozygous (Tubby) pupae occurs in a 


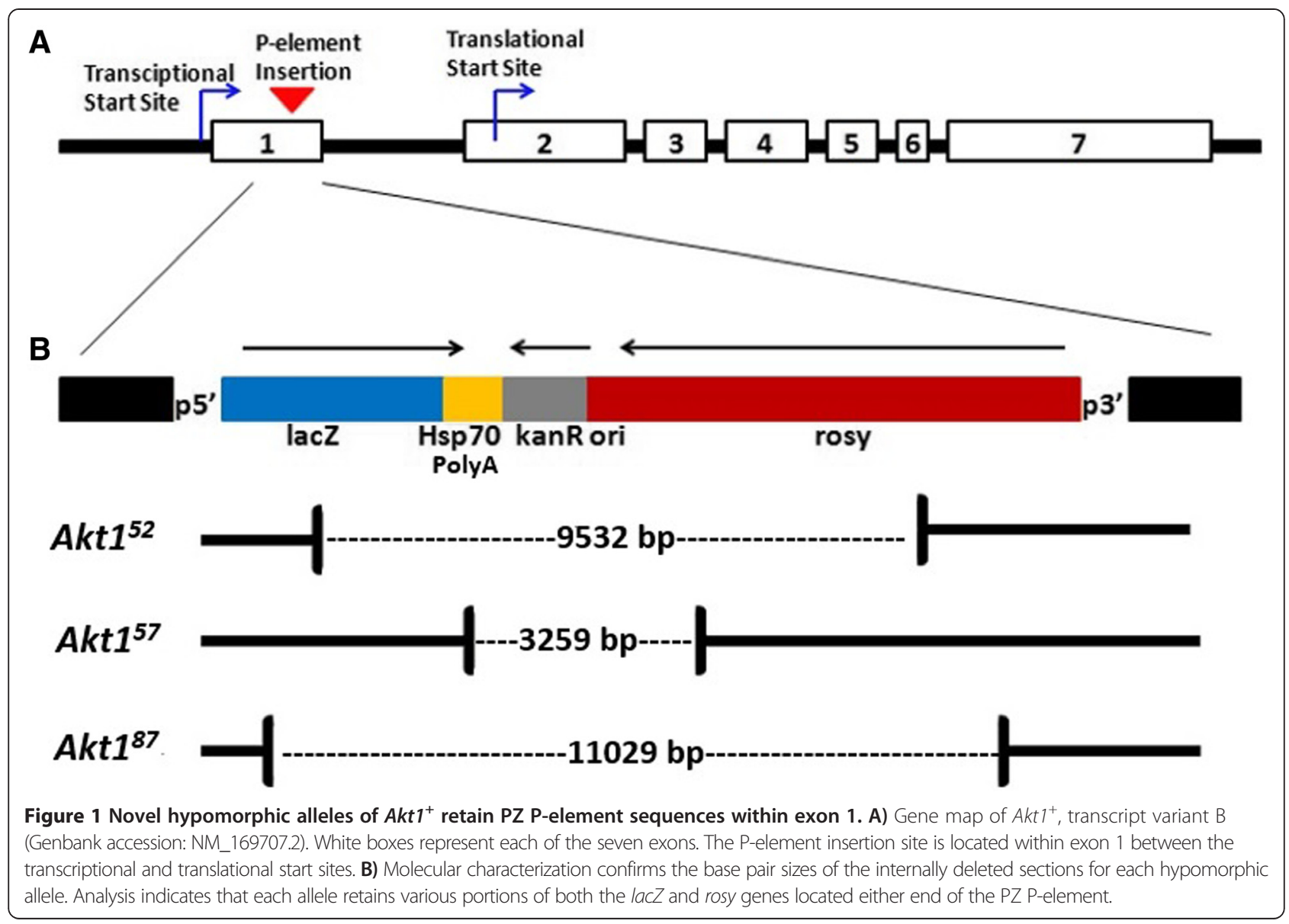

similar time-frame to the control lines (data not shown). The time required to eclose by adult $A k t 1$ heterozygotes (Humeral) and control flies is nine to ten days (Figure 2). Emergence of the homozygous adult flies is delayed by two to four days. One allele, $A k t 1^{87}$, is delayed until day 12 while the other alleles are delayed until day 14 (Figure 2). The extended time of development of homozygotes may be required for the production of the adult mutants.
The eyes of novel Akt1 mutants are reduced in ommatidia size and number

Biometric analysis of homozygous Akt1 mutant eyes indicates there is an overall decrease in both ommatidia number and size when compared to controls (Table 1, Figure 3). The control eyes had a number of $676.4 \pm 13.2$ ommatidia per eye (OPE) and an ommatidia area of $222.6( \pm 4.3) \mathrm{um}^{2}$, which was the largest overall. The original Pelement insertion mutant $A k t 1^{04226}$ has the smallest
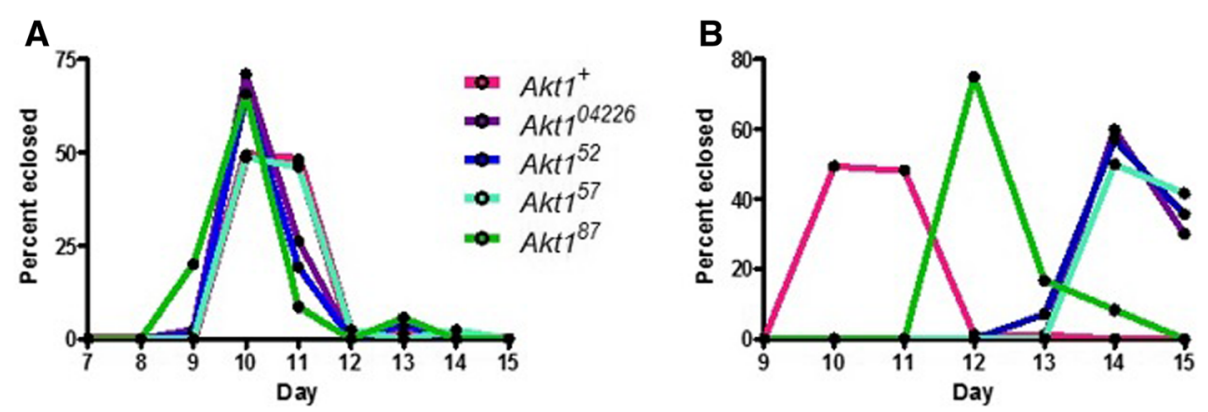

Figure 2 Novel Akt1 mutants are developmentally delayed. Data of developmental delay experiment was plotted as percent eclosed versus day as a line graph. This method allows the peaks of each line to clearly represent the day of which most adult flies eclosed for each genotype.

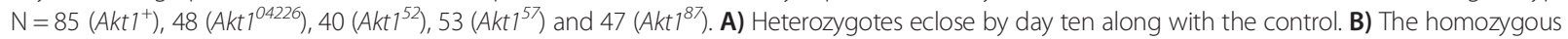
mutants are delayed in growth and do not eclose until two to four days later than the controls. 
Table 1 Biometric analysis of ommatidia area and number in homozygous mutant, transgenic rescue and somatic clones of novel Akt1 mutant alleles

\begin{tabular}{|c|c|c|c|c|c|c|c|c|c|c|}
\hline \multirow[t]{2}{*}{ Allele } & \multicolumn{10}{|c|}{ a) Homozygotes } \\
\hline & $\bar{N}$ & $O A$ & P1 & P2 & P3 & $\mathrm{N}$ & ON & P1 & P2 & P3 \\
\hline \multirow[t]{2}{*}{$A k t 1^{+}$} & 15 & $222.6 \pm 4.3$ & $\mathrm{~N} / \mathrm{A}$ & 0.5286 & $<0.0001$ & 5 & $676.4 \pm 13.2$ & $\mathrm{~N} / \mathrm{A}$ & 0.2179 & 0.1671 \\
\hline & & & & NS & $S$ & & & & NS & NS \\
\hline \multirow[t]{2}{*}{$A k t 1^{04226}$} & 15 & $185.6 \pm 2.4$ & $<0.0001$ & $<0.0001$ & $<0.0001$ & 5 & $604 \pm 2$ & 0.0006 & $<0.0001$ & 0.0007 \\
\hline & & & $\mathrm{S}$ & S & $S$ & & & $S$ & S & $\mathrm{S}$ \\
\hline \multirow[t]{2}{*}{$A k t 1^{52}$} & 15 & $191.1 \pm 2.4$ & $<0.0001$ & 0.0019 & $<0.0001$ & 5 & $544.8 \pm 14.1$ & 0.0001 & $<0.0001$ & $<0.0001$ \\
\hline & & & $\mathrm{S}$ & $S$ & $S$ & & & $S$ & S & $\mathrm{S}$ \\
\hline \multirow[t]{2}{*}{$A k t 1^{57}$} & 15 & $197.6 \pm 3.5$ & 0.0002 & 0.0741 & $<0.0001$ & 5 & $579.4 \pm 11$ & 0.0005 & 0.0057 & 0.0017 \\
\hline & & & $\mathrm{S}$ & NS & $S$ & & & S & $S$ & $\mathrm{~S}$ \\
\hline \multirow[t]{2}{*}{$A k t 1^{87}$} & 15 & $192.8 \pm 2.1$ & $<0.0001$ & $<0.0001$ & $<0.0001$ & 5 & $545.4 \pm 2.5$ & $<0.0001$ & $<0.0001$ & 0.0214 \\
\hline & & & S & S & $S$ & & & S & $S$ & $S$ \\
\hline \multirow[t]{2}{*}{ Allele } & \multicolumn{10}{|c|}{ b) Transgenic Rescues } \\
\hline & $\mathrm{N}$ & OA & P1 & & & $\mathrm{N}$ & ON & P1 & & \\
\hline$\overline{A k t 1^{+}}$ & 15 & $218.9 \pm 2.7$ & $\mathrm{~N} / \mathrm{A}$ & & & 5 & $705.8 \pm 17.6$ & $\mathrm{~N} / \mathrm{A}$ & & \\
\hline \multirow[t]{2}{*}{$A k t 1^{04226}$} & 15 & $204.7 \pm 1.8$ & 0.0002 & & & 5 & $683.2 \pm 3.9$ & 0.2445 & & \\
\hline & & & $\mathrm{S}$ & & & & & NS & & \\
\hline \multirow[t]{2}{*}{$A k t 1^{52}$} & 15 & $206.3 \pm 3.6$ & 0.0086 & & & 5 & $701 \pm 12.6$ & 0.8297 & & \\
\hline & & & $\mathrm{S}$ & & & & & NS & & \\
\hline \multirow[t]{2}{*}{$A k t 1^{57}$} & 15 & $206.2 \pm 3.2$ & 0.0052 & & & 5 & $644.6 \pm 13.5$ & 0.0246 & & \\
\hline & & & $\mathrm{s}$ & & & & & S & & \\
\hline \multirow[t]{2}{*}{$A k t 1^{87}$} & 15 & $212.9 \pm 2.5$ & 0.1112 & & & 5 & $643.6 \pm 7.1$ & 0.0112 & & \\
\hline & & & NS & & & & & S & & \\
\hline \multirow[t]{2}{*}{ Allele } & c) $\mathrm{S}$ & atic Clones & & & & & & & & \\
\hline & $\mathbf{N}$ & OA & P1 & & & $\mathrm{N}$ & ON & P1 & & \\
\hline $\operatorname{Akt}^{+}$ & 9 & $212.1 \pm 7.1$ & $\mathrm{~N} / \mathrm{A}$ & & & 3 & $704.3 \pm 5.5$ & $\mathrm{~N} / \mathrm{A}$ & & \\
\hline \multirow[t]{2}{*}{$A k t 1^{04226}$} & 9 & $179.3 \pm 8.3$ & $<0.0001$ & & & 3 & $508.3 \pm 35.4$ & 0.0007 & & \\
\hline & & & S & & & & & S & & \\
\hline \multirow[t]{2}{*}{$A k t 1^{52}$} & 9 & $148.7 \pm 3.9$ & $<0.0001$ & & & 3 & $261 \pm 14.3$ & $<0.0001$ & & \\
\hline & & & $\mathrm{S}$ & & & & & S & & \\
\hline \multirow[t]{2}{*}{$A k t 1^{57}$} & 9 & $175.7 \pm 9.6$ & $<0.0001$ & & & 3 & $483 \pm 24.5$ & 0.0001 & & \\
\hline & & & $\mathrm{S}$ & & & & & S & & \\
\hline \multirow[t]{2}{*}{$A k t 1^{87}$} & 9 & $173.8 \pm 7.9$ & $<0.0001$ & & & 3 & $501 \pm 33.2$ & 0.0005 & & \\
\hline & & & S & & & & & S & & \\
\hline
\end{tabular}

$\mathrm{OA}=$ Ommatidia Area $\left(\mathrm{um}^{2}\right), \mathrm{ON}=$ Ommatidia Number, $\mathrm{P} 1=\mathrm{P}$-value when compared to $A k t 1^{+}$control, $\mathrm{P} 2=\mathrm{P}$-value when compared to transgenic rescue counterpart, P3 = P-value when compared to somatic clone counterpart, $\mathrm{S}=$ significant, NS = not significant.

ommatidia area of $185.6 \pm 2.4 \mathrm{um}^{2}$, but with an ommatidia number of $604 \pm 2$ OPE. The three novel Akt1 hypomorphs were all smaller than the control in ommatidia area and significantly reduced in ommatidia number when compared to both the control and the original P-element insertion mutant. Of these three, $A k t 1^{52}$ is the smallest with a count of $544.8 \pm 14.1$ OPE and an area of $191.1 \pm$ $2.4 \mathrm{um}^{2}$. The mutant $A k t 1^{87}$ is slightly larger with an ommatidia area of $192.8 \pm 2.1 \mathrm{um}^{2}$ and $545.4 \pm 2.5$ OPE.
The largest of the mutants is $A k t 1^{57}$ with an ommatidia area of $197.6 \pm 3.5 \mathrm{um}^{2}$ and an ommatidia number of $579.4 \pm 11$ OPE.

\section{Transgenic replacement partially rescue the phenotype of} mutant homozygotes

Ubiquitous expression of wild-type Akt1 in the background of the homozygous mutants results in a partial rescue of both ommatidia size and number (Table 1, 


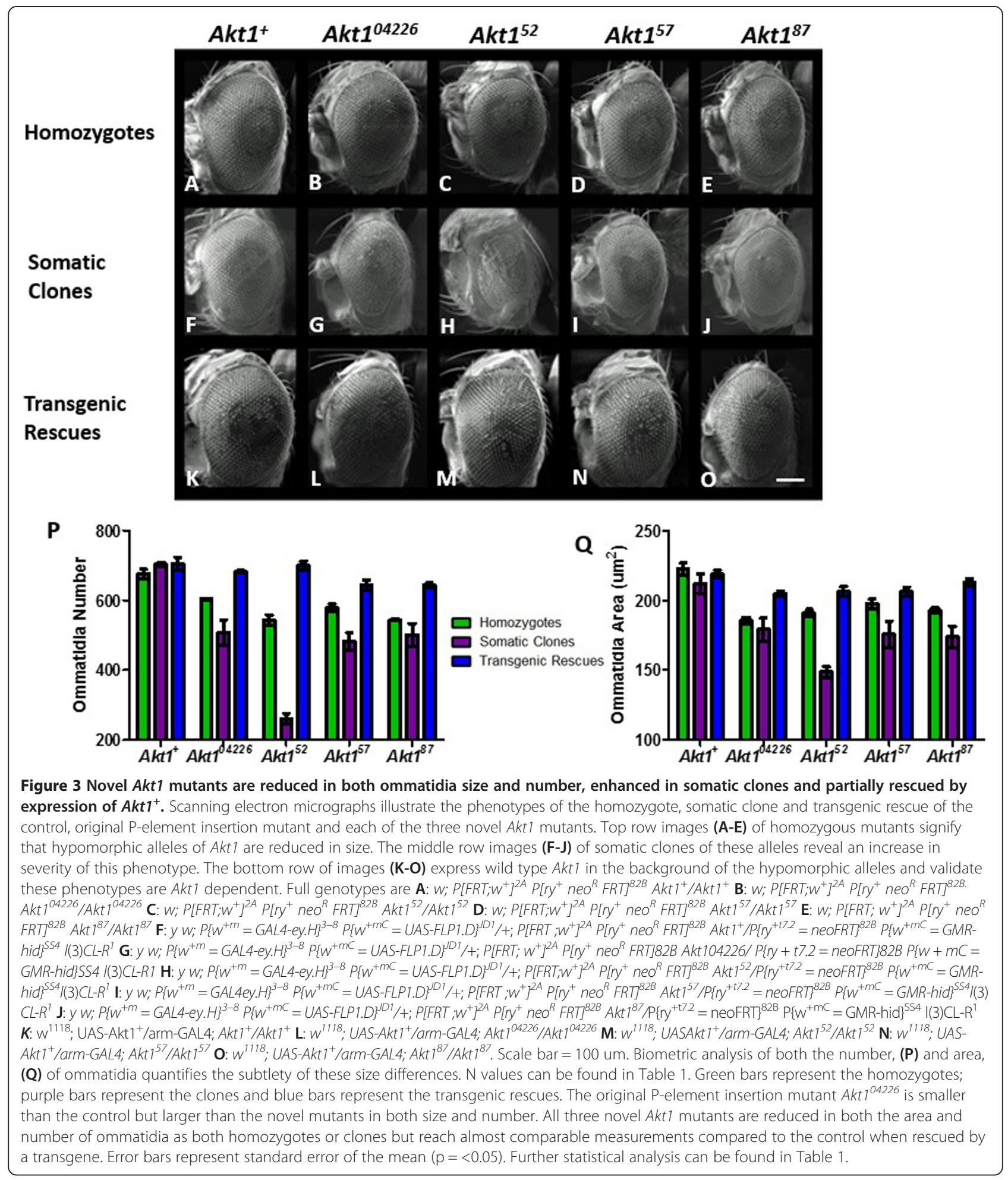

Figure 3). The transgenic control $A k t 1^{+}$is the largest eye overall having an ommatidia area of $218.9 \pm 2.7 \mathrm{um}^{2}$ and a total number of $705.8 \pm 17.6$ OPE. The transgenic expression of $A k t 1^{+}$in the background of the original P-element insertion mutant results in eyes that are only slightly smaller than the control having an ommatidia area of $204.7 \pm 1.8 \mathrm{um}^{2}$ and a total of $683.2 \pm 3.9$ OPE. In all cases the size of the ommatidia and the total count of ommatidia for the mutants with transgenic replacement of wild-type $A k t 1^{+}$does not differ significantly from the 
control (Figure 3). The average ommatidia area is very similar for $A k t 1^{52}$ and $A k t 1^{57}$ being $206.3 \pm 3.6 \mathrm{um}^{2}$ and $206.2 \pm 3.2 \mathrm{um}^{2}$ respectively; while the area for $A k t 1^{87}$ is larger at $212.9 \pm 2.5 \mathrm{um}^{2}$. The ommatidia number for the partially rescued mutants is similar for $A k t 1^{57}$ and $A k t 1^{87}$ being $644.6 \pm 13.5 \mathrm{OPE}$ and $643.6 \pm 7.1 \mathrm{OPE}$ respectively, with $A k t 1^{52}$ having a few more ommatidia at $701 \pm 12.6 \mathrm{OPE}$.

\section{Somatic clones of the eye have a more severe phenotype than the homozygotes}

Given the developmental delay of the Akt1 homozygotes, analysis of somatic clones of the eye was carried out. The FLP recombinase was driven by the eyeless promoter to direct expression in the developing eye tissue. In the presence of FLP, homologous chromosomes undergo mitotic recombination between the FRT sites located on chromosome pairs. Heterozygous parent cells can produce both homozygous Akt1 mutant cells containing two copies of the mutant allele, and cells containing two copies of $A k t 1^{+}$. In this system, the $A k t 1^{+}$ daughter cells are lost due to the presence of a linked recessive cell lethal mutation located on the same arm of the chromosome bearing the $A k t 1^{+}$allele. Thus, in the eyes of clone bearing flies, the surviving cells bear two copies of the Akt1 mutant allele under investigation. The clone of the control is the largest and most consistent in size when compared to its homozygous and transgenic rescued counterpart having an ommatidia area of 212.1 $\pm 7.1 \mathrm{um}^{2}$ and number of $704.3 \pm 5.5$ OPE. The cloned original P-element insertion mutant $A k t 1^{04226}$ is reduced in both size and number compared to its original homozygous version with an average area of $179.3 \pm 8.3 \mathrm{um}^{2}$ and ommatidia number of $508.3 \pm 35.4 \mathrm{OPE}$, yet is comparable in size and number to two of the cloned novel mutants, $A k t 1^{57}$ and $A k t 1^{87}$, which have an ommatidia area of $175.7 \pm 9.6 \mathrm{um}^{2}$ and $173.8 \pm 7.9 \mathrm{um}^{2}$ and a count of $483 \pm 24.5$ OPE and $501 \pm 33.2$ OPE respectively. The measurement and count of ommatidia for both of these mutants is significantly smaller than that of their homozygous versions. Of all the mutants, $A k t 1^{52}$ exhibits the most severe phenotype with the greatest decrease in ommatidia area $\left(148.7 \pm 3.9 \mathrm{um}^{2}\right)$ and number $(261 \pm 14.3$ OPE) when compared to both its homozygous counterpart as well as with the other cloned mutants.

Akt1-foxo double mutant lines reveal an epistatic relationship Drosophila lines having both the novel Akt1 mutant alleles in combination with a null foxo mutant allele resemble the original foxo mutant more closely than the Akt1 mutants (Figure 4; Table 2). The controls OrR and $w^{1118}$ have an ommatidia area of $189.4 \pm 1.43 \mathrm{um}^{2}$ and $185.5 \pm 1.34 u^{2}{ }^{2}$ and an ommatidia count of $675.7 \pm 4.4$ OPE and $665.3 \pm 9.6$ OPE respectively. In comparison, the null foxo mutant eye has a smaller average ommatidia area of $170.4 \pm 1.42 \mathrm{um}^{2}$ and a higher ommatidia count of $723 \pm 5.6$ OPE. The double mutant lines are smaller than both the controls and the null foxo mutant in size of ommatidia, but have counts of ommatidia that are not significantly different from the null foxo mutant. Both the double mutants bearing $A k t 1^{04226}$ and $A k t 1^{57}$ alleles have similar ommatidia areas of $154.4 \pm 1.2 \mathrm{um}^{2}$ and $156.3 \pm 1.1 \mathrm{um}^{2}$, and ommatidia numbers of $735 \pm$ 5.2 OPE and $733.9 \pm 6.3$ OPE respectively. The double mutant bearing the $A k t 1^{52}$ allele is closer to the null foxo mutant in ommatidia size $\left(166.3 \pm 1.7 \mathrm{um}^{2}\right)$ but has slightly fewer ommatidia with $709.1 \pm 8.2$ OPE. The ommatidia size for each of the double mutants is considerably smaller than the original homozygous mutant versions of each Akt1 mutant allele, while the counts of ommatidia are much higher, exhibiting the same trend as the null foxo mutant in comparison to the novel Akt1 mutants.

\section{Discussion}

Viable novel Akt1 hypomorphs were generated via imprecise P-element excision and were found to retain internally deleted versions of the original $P Z$ P-element upstream of the Akt1 gene's protein coding region (Figure 1). Three selected $A k t 1$ hypomorphs were characterized phenotypically as small in size and delayed in terms of developmental time. In Drosophila, the development from egg to adult involves three larval stages plus pupation before the non-growing sexually mature adult fly arises. The timing of transition between these stages is dependent upon the rate of growth. The insulin receptor signalling pathway is a major contributor in the control of growth and has been implicated in the control of the onset of metamorphosis in Drosophila [28]. Ablation of insulin producing cells within the larval brain decreases the growth rate and delays metamorphosis in Drosophila, as does a loss-of-function mutation of the insulin receptor [29,30]. As Akt1 is a central component of the insulin receptor signalling pathway, it is not surprising that these novel hypomorphic alleles result in a delay of development and overall smaller adult organisms.

Due to the extension in the time for the novel mutants to undergo eclosion, a comparison of the phenotypes of the eye for both homozygous mutants and somatic clones was undertaken. The clone eyes are comprised of homozygous mutant tissue in a heterozygous organism that develops within a relatively normal timeframe. Biometric analysis of the eyes of these mutant clones revealed enhanced severity of the decreased growth phenotype. Adult organisms, as well as their organs and tissues, have a tendency to develop within a range of normal overall size, such that the cellular composition may vary from a large number of small cells, to a small 


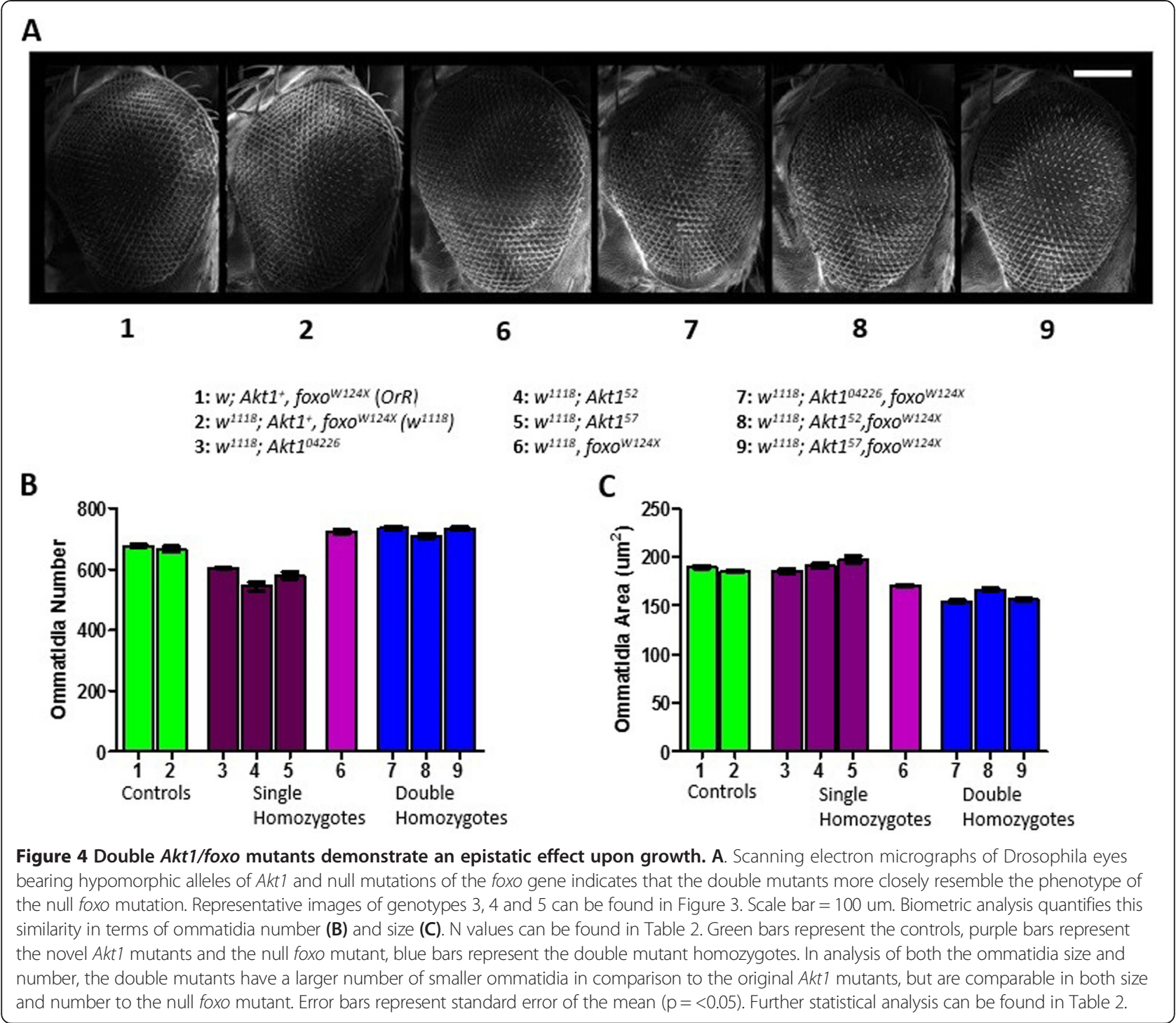

Table 2 Biometric analysis of ommatida area and number of Drosophila eyes bearing both a novel Akt1 mutant allele and a null foxo mutant allele

\begin{tabular}{|c|c|c|c|c|c|c|c|c|c|c|c|c|}
\hline Genotype & $\mathbf{N}$ & OA & P1 & P2 & P3 & P4 & $\mathbf{N}$ & ON & P1 & P2 & P3 & P4 \\
\hline$w^{+} ; A_{k t 1}{ }^{+}$foxo ${ }^{+}$(OrR) & 48 & $189.4 \pm 1.4$ & $\mathrm{~N} / \mathrm{A}$ & $\mathrm{N} / \mathrm{A}$ & $\mathrm{N} / \mathrm{A}$ & $\mathrm{N} / \mathrm{A}$ & 16 & $675.7 \pm 4.4$ & $\mathrm{~N} / \mathrm{A}$ & $\mathrm{N} / \mathrm{A}$ & $\mathrm{N} / \mathrm{A}$ & $\mathrm{N} / \mathrm{A}$ \\
\hline$w^{1118} ; A_{k t 1^{+}}$foxo ${ }^{+}\left(w^{1118}\right)$ & 36 & $185.5 \pm 1.3$ & $\mathrm{~N} / \mathrm{A}$ & $\mathrm{N} / \mathrm{A}$ & $\mathrm{N} / \mathrm{A}$ & $\mathrm{N} / \mathrm{A}$ & 12 & $665.3 \pm 9.6$ & $\mathrm{~N} / \mathrm{A}$ & $\mathrm{N} / \mathrm{A}$ & $\mathrm{N} / \mathrm{A}$ & $\mathrm{N} / \mathrm{A}$ \\
\hline \multirow[t]{2}{*}{$w^{1118} ; A_{k t 1^{+}}$foxo ${ }^{W 124 X}$} & 42 & $170.4 \pm 1.4$ & $<0.0001$ & $<0.0001$ & N/A & $\mathrm{N} / \mathrm{A}$ & 14 & $723.0 \pm 5.6$ & $<0.0001$ & $<0.0001$ & $\mathrm{~N} / \mathrm{A}$ & $\mathrm{N} / \mathrm{A}$ \\
\hline & & & $\mathrm{S}$ & $S$ & & & & & $S$ & $S$ & & \\
\hline \multirow[t]{2}{*}{$w^{1118} ; A k t 1^{04226}$ foxo ${ }^{W 124 X}$} & 45 & $154.4 \pm 1.2$ & $<0.0001$ & $<0.0001$ & $<0.0001$ & $<0.0001$ & 15 & $735.0 \pm 5.2$ & $<0.0001$ & $<0.0001$ & $<0.0001$ & 0.1282 \\
\hline & & & $S$ & $S$ & S & S & & & S & S & S & NS \\
\hline \multirow[t]{2}{*}{$w^{1118} ; A k t 1^{52}$ foxo ${ }^{W 124 X}$} & 39 & $166.3 \pm 1.7$ & $<0.0001$ & $<0.0001$ & $<0.0001$ & 0.0630 & 13 & $709.1 \pm 8.2$ & 0.0008 & 0.0020 & $<0.0001$ & 0.1686 \\
\hline & & & S & S & $S$ & NS & & & S & S & $S$ & NS \\
\hline \multirow[t]{2}{*}{$w^{1118} ;$ Akt $^{57}$ foxo ${ }^{W 124 X}$} & 54 & $156.3 \pm 1.1$ & $<0.0001$ & $<0.0001$ & $<0.0001$ & $<0.0001$ & 18 & $733.9 \pm 6.3$ & $<0.0001$ & $<0.0001$ & $<0.0001$ & 0.2212 \\
\hline & & & $\mathrm{S}$ & $S$ & $S$ & S & & & $S$ & $\mathrm{~S}$ & $\mathrm{~S}$ & NS \\
\hline
\end{tabular}

OA $=$ Ommatidia Area $\left(\mathrm{um}^{2}\right), \mathrm{ON}=$ Ommatidia Number, $\mathrm{P} 1=\mathrm{P}$-value when compared to the Oregon $R(O r R)$ control, $\mathrm{P} 2=\mathrm{P}$-value when compared to the $W$ NS $=$ not significant. 
number of large cells. Cell growth includes an increase in cell number and cell size, and while not mutually exclusive, both can be regulated by distinct extracellular processes [11,31-33], including the insulin receptor signalling pathway, which is highly conserved between invertebrates and mammals [34]. Reduced expression or loss of $A k t 1$, the central component of insulin receptor signalling, can result in the production of smaller animals or, if severe, lethality $[35,16,15]$. The smaller eyes observed in the homozygotes is expected with the lower expression of $A k t 1$ in these novel mutants.

Compensatory growth is widespread and occurs in the surviving cells of damaged tissues to generate final structures of near normal overall size [36,37]. This growth consists of remodeling the existing tissue to regenerate the full body plan in response to tissue damage leading to the development of a smaller but still complete and intact organism. In order to maintain tissue homeostasis, cells that survive the tissue damage can compensate for those that are lost by increasing their rate of proliferation and cell divisions. Cells in Drosophila that have experienced an increase in cell death via radiation showed an increase in proliferation by the surviving cells [38]. Compensatory proliferation has been shown to lead to the development of normal-sized adult wings even when $40-60 \%$ of cells in the wing disc of Drosophila are either killed or rendered incapable of further proliferation [39]. These novel Akt1 hypomorphs have been shown to be developmentally delayed and result in the formation of small adult flies. The mutant clone eyes show a more severe phenotype due to the reduced replacement of missing tissue without the extended time during development. Clearly, the extended period of time required for these mutants to develop allows compensatory proliferation to generate smaller but intact adults.

In order to begin to understand the mechanisms responsible for the observed compensatory growth, double mutant lines of the novel Akt1 hypomorphs and an amorphic allele of foxo, a gene encoding a key downstream target of $A k t 1$, were generated. The transcription factor foxo is known as a major effector of insulin receptor signalling and has been implicated in the control of cell growth. Overexpression of the mammalian homologues of foxo, as well as Drosophila foxo, leads to growth arrest $[7,40]$ which can be suppressed with increased insulin receptor signalling. This suppression is ineffective when the foxo transcription factor has been made incapable of phosphorylation, and thus nuclear exclusion, by Akt1. In addition to this, foxo governs the expression of target genes that encode factors that regulate cell growth such as the eukaryotic initiation factor 4Ebinding protein (4E-BP) gene and cell cycle regulators including $\mathrm{p} 27^{\mathrm{kip} 1}$. The $4 E-B P$ product is a negative regulator of protein synthesis and has been shown to strongly influence the regulation of cell growth [41]. When foxo is upregulated, so is $4 \mathrm{E}-\mathrm{BP}$, which binds to the messenger RNA 5' cap-binding protein eIF4E to inhibit protein synthesis and cell growth. In humans, p2 $7^{\text {kip1 }}$ inhibits cyclindependent kinases (cdks) [42], which aid in promoting the transitions between cell-cycle phases. Overexpression of p2 $7^{\mathrm{kip} 1}$ in human cells leads to cell-cycle arrest in the G1 phase, and when foxo and, subsequently, $p 27^{k i p 1}$, is upregulated $[41,43]$. Co-expression of foxo and constitutively active Ras2, which can induce G1/S progression and cell proliferation, is able to partially rescue the phenotype in the eye that is observed with an overexpression of foxo alone [7]. An increase in foxo activity appears to result in a decrease in cell proliferation. The double mutants, having both hypomorphic alleles of $A k t 1$ and null alleles of foxo, more closely resemble the foxo mutants. Analysis of ommatidia number shows an epistatic effect whereas an argument could be made in the comparison of ommatidia area for a slight synergistic enhancement of the phenotype. Regardless this suggests that without the presence of the foxo gene product, the hypomorphic alleles of $A k t 1$ do not cause the same reduction of growth and strongly suggests that foxo is necessary for the processes that lead to compensatory growth.

\section{Conclusions}

Through the generation of clones, we were able to uncover a more severe effect of these Akt1 hypomorphs upon the control of growth. Originally, the generation of somatic clones was utilized to study homozygous tissue in a heterozygous organism when the homozygotes themselves were not viable. However, when used to study homozygous tissues of viable, yet small and developmentally delayed organisms, this system can expose a subtle phenotype previously obscured by compensatory proliferation. Developmental delay is a common phenomenon associated with many genetic mutations and could potentially play a significant role in the final phenotype. Generation of somatic clones would eliminate this developmental timing factor, thereby clarifying the impact a genetic mutation has on cellular processes including growth.

\section{Animal ethics}

This study was conducted under the approval of the Animal Care Committee of Memorial University of Newfoundland as a Category of Invasiveness Level A protocol under the project title of "Genetic, biochemical and molecular analysis of cell survival and cell death in Drosophila melanogaster" (protocol number: 14-09-BS).

\section{Abbreviations}

foxo: Forkhead box subgroup "O"; Ilp: Insulin-like peptide; InR: Insulin receptor; OPE: Ommatidia per eye.

Competing interests

The authors declare that they have no competing interests. 


\section{Authors' contributions}

JDS generated some recombinant lines, performed the molecular characterizations, the developmental timing evaluations, the scanning electron microscopy and the biometric analysis of the mutants, performed the statistical analyses and drafted the initial manuscript. BES generated the mutants and some recombinant lines, conceived and participated in the design and supervision of the study and contributed significantly to the final draft of the manuscript. Both authors have read and approved the final manuscript.

\section{Acknowledgments}

The authors would like to thank Drs. Liqui Men, formerly of the Bio-molecular Imaging Cluster at Memorial University of Newfoundland, and Michael Shaffer, formerly of the Microanalysis facility of Memorial University of Newfoundland for technical assistance with scanning electron microscopy. Thanks to members of the Genomics and Proteomics facility at Memorial University of Newfoundland for assistance with sequencing. Thanks also to Drs. Martin Junger and Ernst Hafen for providing the foxo ${ }^{\text {W124X }}$ line, Dr. Howard Lipshitz for providing the $w^{1718}$ line, and to Dr. Norbert Perrimon for providing the original $w^{1118} ;$ P[FRT; $\left.W^{+}\right]^{2 A} P\left[r y^{+} n e o^{R}\right.$ $F R]^{82 B}$ line. Finally, we thank the anonymous reviewers for very helpful comments and criticisms. This project was funded by a Natural Sciences and Engineering Research Council of Canada (NSERC) Post-Graduate Scholarship to JDS and NSERC Discovery Grants to BES. JDS was also partially funded by a Department of Biology Teaching Assistantship and a School of Graduate Studies Fellowship from Memorial University of Newfoundland.

Received: 25 November 2014 Accepted: 24 February 2015 Published online: 11 March 2015

\section{References}

1. Robertson CW. The metamorphosis of Drosophila melanogaster, including an accurately timed account of the principal morphological changes. J Morphol. 1936;59:351-99.

2. Beadle GW, Anderson RL, Maxwell J. A comparison of the diffusible Substances Concerned with Eye Color Development in Drosophila, Ephestia and Habrobracon. Proc Natl Acad Sci U S A. 1938:24(2):80-5.

3. Tennessen JM, Thummel CS. Coordinating Growth and Maturation - Insights from Drosophila. Curr Biol. 2011;21:R750-7.

4. Tatar M. Diet restriction in Drosophila melanogaster. Design and analysis. Interdiscip Top Gerontol. 2007;35:115-36.

5. Mirth CK, Riddiford LM. Size assessment and growth control: how adult size is determined in insects. Bioessays. 2007;29(4):344-55.

6. Clancy DJ, Gems D, Hafen E, Leevers SJ, Partridge L. Dietary restriction in long-lived dwarf flies. Science. 2002;296(5566):319.

7. Kramer JM, Davidge JT, Lockyer JM, Staveley BE. Expression of Drosophila FOXO regulates growth and can phenocopy starvation. BMC Dev Biol. 2003:3:5.

8. Arking R. Multiple longevity phenotypes and the transition from health to senescence. Ann N Y Acad Sci. 2005;1057:16-27.

9. Geminard C, Arquier N, Layalle S, Bourouis M, Slaidina M, Delanoue R, et al. Control of metabolism and growth through insulin-like peptides in Drosophila. Diabetes. 2006:55 Suppl 2:S5-8.

10. Oldham S, Bohni R, Stocker H, Brogiolo W, Hafen E. Genetic control of size in Drosophila. Phil Trans R Soc Lond B. 2000;355:945-52.

11. Coelho CM, Leevers SJ. Do growth and cell division rates determine cell size in multicellular organisms? J Cell Sci. 2000;113(Pt 17):2927-34.

12. Aoyama H, Daitoku H, Fukamizu A. Nutrient control of phosphorylation and translocation of Foxo 1 in C57BL/6 and db/db mice. Int J Mol Med. 2006;18(3):433-9.

13. Edgar BA. How flies get their size: genetics meets physiology. Nat Rev Genet. 2006;7(12):907-16.

14. Wu Q, Brown MR. Signaling and function of insulin-like peptides in insects. Annu Rev Entomol. 2006:51:1-24.

15. Verdu J, Buratovich MA, Wilder EL, Birnbaum MJ. Cell-autonomous regulation of cell and organ growth in Drosophila by Akt/PKB. Nat Cell Biol. 1999; (8):500-6.

16. Staveley BE, Ruel L, Jin J, Stambolic V, Mastronardi FG, Heitzler P, et al. Genetic analysis of protein kinase B (AKT) in Drosophila. Curr Biol. 1998;8(10):599-602.

17. Burgering BM, Kops GJ. Cell cycle and death control: long live Forkheads. Trends Biochem Sci. 2002;27(7):352-60.
18. Barthel A, Schmoll D, Unterman TG. FoxO proteins in insulin action and metabolism. Trends Endocrinol Metab. 2005;16(4):183-9.

19. Puig O, Tjian R. Transcriptional feedback control of insulin receptor by dFOXO/FOXO1. Genes Dev. 2005:19(20):2435-46.

20. Gershman B, Puig O, Hang L, Peitzsch RM, Tatar M, Garofalo RS. Highresolution dynamics of the transcriptional response to nutrition in Drosophila: a key role for dFOXO. Physiol Genomics. 2007;29(1):24-34.

21. Gao X, Neufeld TP, Pan D. Drosophila PTEN regulates cell growth and proliferation through PI3K-dependent and -independent pathways. Dev Biol. 2000;221(2):404-18.

22. Stocker $H$, Andjelkovic M, Oldham S, Laffargue M, Wymann MP, Hemmings BA, et al. Living with lethal PIP3 levels: viability of flies lacking PTEN restored by a PH domain mutation in Akt/PKB. Science. 2002;295(5562):2088-91.

23. Lee $\mathrm{G}$, Chung J. Discrete functions of rictor and raptor in cell growth regulation in Drosophila. Biochem Biophys Res Commun. 2007;357(4):1154-9.

24. Robertson HM, Preston CR, Phillis RW, Johnson-Schlitz DM, Benz WK, Engels WR. A stable genomic source of P element transposase in Drosophila melanogaster. Genetics. 1988;118(3):461-70.

25. Junger MA, Rintelen F, Stocker H, Wasserman JD, Vegh M, Radimerski T, et al. The Drosophila forkhead transcription factor FOXO mediates the reduction in cell number associated with reduced insulin signaling. J Biol. 2003;2(3):20.

26. Stowers RS, Schwarz TL. A genetic method for generating Drosophila eyes composed exclusively of mitotic clones of a single genotype. Genetics. 1999;152(4):1631-9.

27. Abramoff MD, Magelhaes PJ, Ram SJ. Image Processing with ImageJ. Biophot Int. 2004;11(7):36-42.

28. Walkiewicz MA, Stern M. Increased Insulin/Insulin Growth Factor Signaling Advances the Onset of Metamorphosis in Drosophila. PLoS. 2009;4(4):e5072.

29. Rulfison EJ, Kim SK, Nusse R. Ablation of insulin-producing neurons in flies: growth and diabetic phenotypes. Science. 2002;296(5570):1118-20.

30. Shingleton AW. Body-size regulation: combining genetics and physiology. Curr Biol. 2005;15(20):R825-7.

31. Day SJ, Lawrence PA. Measuring dimensions: the regulation of size and shape. Dev (Cambridge, England). 2000;127(14):2977-87.

32. Stocker H, Hafen E. Genetic control of cell size. Curr Opin Genet Dev. 2000;10(5):529-35.

33. Johnston LA, Gallant P. Control of growth and organ size in Drosophila. Bioessays. 2002;24(1):54-64.

34. Brogiolo W, Stocker H, Ikeya T, Rintelen F, Fernandez R, Hafen E. An evolutionarily conserved function of the Drosophila insulin receptor and insulin-like peptides in growth control. Curr Biol. 2001;11(4):213-21.

35. Kennedy SG, Wagner AJ, Conzen SD, Jordan J, Bellacosa A, Tsichlis PN, et al. The PI3-kinase/Akt signaling pathway delivers an anti-apoptotic signal. Genes Dev. 1997;11(6):701-13.

36. Gerhold AR, Richter DJ, Yu AS, Hariharan IK. Identification and Characterisation of Genes Required for Compensatory Growth in Drosophila. Genetics. 2011;189:1309-26.

37. Worley MI, Setiawa L, Hariharan IK. Regeneration and Transdetermination in Drosophila Imaginal Discs. Annu Rev Genet. 2012;46:289-310.

38. Schweizer P. Effect of X-rays on the development of male genital primordia in Drosophila melanogaster and investigation of recovery by cell clone analysis. Biophysik. 1972;8:158-88.

39. Haynie $J$, Bryant PJ. The effects of X-rays on the proliferation dynamics of cells in the wing discs of Drosophila melanogaster. Dev Genes Evol. 1977:183:85-100

40. Neufeld TP. Shrinkage control: regulation of insulin-mediated growth by FOXO transcription factors. J Biol. 2003;2:18.

41. Miron M, Verdu J, Lachance PE, Birnbaum MJ, Lasko PF, Sonenberg N. The translational inhibitor 4E-BP is an effector of $\mathrm{PI}(3) \mathrm{K} / \mathrm{Akt}$ signalling and cell growth in Drosophila. Nat Cell Biol. 2001;3(6):596-601.

42. Dijkers PF, Medema RH, Pals C, Banerji L, Thomas NS, Lam EW, et al. Forkhead transcription factor FKHR-L1 modulates cytokine-dependent transcriptional regulation of p27(KIP1). Mol Cell Biol. 2000;20(24):9138-48.

43. Van Der Heide LP, Hoekman MF, Smidt MP. The ins and outs of FoxO shuttling: mechanisms of FoxO translocation and transcriptional regulation. Biochem J. 2004:380(Pt 2):297-309. 